\title{
Análisis de la capacidad de respuesta de los sistemas de información en salud para la supervisión de riesgos que afectan el derecho a la salud en Colombia
}

\author{
Analysis of the Response Capacity of the Health Information Systems to Oversee the Risks Affecting the Right \\ to Health in Colombia
}

Análise da capacidade de resposta dos sistemas de informação em saúde para a supervisão de riscos que afetam o direito à saúde na Colômbia

Julia Isabel Eslava-Rincón ${ }^{\mathrm{a}}$

Instituto de Salud Pública, Pontificia Universidad

Javeriana, Colombia

jieslava@javeriana.edu.co

ORCID: http://orcid.org/0000-0001-5644-2973

Fredy Armindo Camelo-Tovar

Instituto de Salud Pública, Pontificia Universidad

Javeriana, Colombia

ORCID: http://orcid.org/0000-0003-4920-3864

Lucía Mina-Rosero

Instituto de Salud Pública, Pontificia Universidad

Javeriana, Colombia

ORCID: http://orcid.org/0000-0002-3886-036X

María Elizabeth Vásquez-Candia

Instituto de Salud Pública, Pontificia Universidad

Javeriana, Colombia

ORCID: http://orcid.org/0000-0001-9603-8916

Marino Mauricio Mejía-Rocha

Instituto de Salud Pública, Pontificia Universidad

Javeriana, Colombia

ORCID: http://orcid.org/0000-0003-0769-2612
DOI: https://doi.org/10.11144/Javeriana.rgps17-35.acrs Redalyc: http://www.redalyc.org/articulo.oa?id=54557477007

Fecha de recepción: 12 Diciembre 2017 Fecha de aprobación: 14 Marzo 2018 Fecha de publicación: 01 Noviembre 2018

\section{Resumen:}

Este artículo tiene por objeto evidenciar la capacidad de respuesta de los sistemas de información en salud (SIS) en Colombia para soportar un modelo de inspección, vigilancia y control (IVC) orientado al seguimiento de los riesgos que menoscaban el acceso, el uso y la calidad de los servicios de salud. Se realizó un estudio transversal, descriptivo, desde el enfoque de gestión de riesgos, que incluyó una revisión del marco regulatorio de las amenazas para el goce efectivo del derecho (GED) a la salud y las características de la información reportada por los actores del sistema, requeridas para su seguimiento. Los datos que soportan el seguimiento a los procesos de atención y su impacto sobre la salud poblacional resultan insuficientes para hacer una adecuada supervisión de los riesgos. Se deben aprovechar los cambios que experimenta actualmente el sistema de salud para replantear la forma en que su desempeño es objeto de seguimiento.

Palabras clave: sistemas de información en salud, legislación sanitaria, derecho a la salud, gestión de riesgos, monitoreo, sistemas de salud. 


\begin{abstract}
:
This article aims to disclose the response capacity of the Health Information Systems (HIS) in Colombia to support an inspection, s urveillance and control model (ISC) driven towards the monitoring of the risks undermining the access, use and quality of the health services. This paper is based on a descriptive cross-sectional study under a risk management approach including a review of the regulations framework dealing with the threats to the effective enjoyment of the right to health (EERH) and the characteristics of the information reported by the actors in the system, which is required to carry out the said monitoring. The data supporting the monitoring to the health care processes and its impact on the population health are not enough to appropriately oversee the risks involved. Advantage should be taken from the changes currently taking place in the health system in order to rethink how the system performance is taken as monitored object. Keywords: health information systems, public health regulations, right to health, risk management, monitoring, health systems.
\end{abstract}

\title{
Resumo:
}

Este artigo objetiva evidenciar a capacidade de resposta dos sistemas de informação em saúde (SIS) na Colômbia para suportar um modelo de inspeção, vigilância e controle (IVC) orientado ao seguimento dos riscos que prejudicam o acesso, o uso e a qualidade dos serviços de saúde. Realizou-se um estudo transversal, descritivo, desde a abordagem de gestão de riscos, que incluiu uma revisão do quadro regulatório das ameaças para o desfrute efetivo do direito (GED) à saúde e as características da informação reportada pelos atores do sistema, requeridas para seu seguimento. Os dados que suportam o seguimento aos processos de atenção e seu impacto sobre a saúde populacional resultam insuficientes para fazer uma adequada supervisão dos riscos. Devem-se aproveitar as mudanças que experimenta atualmente o sistema de saúde para recolocar a forma em que seu desempenho é objeto de seguimento.

Palavras-chave: sistemas de informação em saúde, legislação sanitária, direito à saúde, gestão de riscos, monitoramento, sistemas de saúde.

\section{Introducción}

Las reformas al Estado promovidas en los años ochenta y noventa propendían por una gestión pública regida por la eficiencia, la privatización, la tercerización de los servicios sociales y la introducción de la lógica del mercado para el logro de sus finalidades. En consecuencia, el Estado dejó de ser interventor, productor y prestador de servicios, para fortalecer sus capacidades regulatorias y de formulación de políticas, establecer las reglas de juego para la entrada de agentes privados y otorgar o compartir responsabilidades en la prestación de servicios sociales (1). Estas reformas tocaron sensiblemente al sistema de salud colombiano, que con la promulgación de la Ley 100 de 1993 acogió en su seno la lógica del mercado, delegando en el Estado la función de dirigir, orientar, regular, controlar y vigilar la prestación de los servicios de salud (2).

Sin embargo, estas reformas no pudieron ser implementadas plenamente en el contexto colombiano y tampoco lograron enfrentar la crisis suscitada por la desmejora en las condiciones de vida y el empobrecimiento de la población que se intensificó en la segunda mitad de los noventa. En consecuencia, han emergido nuevas olas que, sin abandonar los preceptos de la primera, se han enfocado en recuperar el protagonismo del Estado y corregir las fallas del mercado (3). En este contexto, con la apuesta por la reducción de la pobreza y la promoción de un desarrollo más equitativo, se fijaron los Objetivos de Desarrollo del Milenio (ODM-2000-2015), que impusieron nuevos retos a los sistemas de salud, exigiendo al Estado enfocarse en su fortalecimiento, para garantizar el acceso y la mejora de las condiciones de salud de la población (4).

Así, el seguimiento al desempeño de los sistemas de salud se centró en evaluar el efecto de la atención sobre los resultados para la salud de las poblaciones, reivindicando, entre otros factores, un papel más activo del Estado para coordinar, regular y supervisar las acciones de los agentes públicos y privados involucrados en la prestación. Este cometido, por supuesto, requiere sistemas de información en salud eficaces para la producción de información confiable y oportuna, asegurando su uso, análisis y difusión, así como de sistemas de indicadores para el monitoreo, el seguimiento y la evaluación que soporten la toma de decisiones que garanticen el acceso, el uso y la calidad de los servicios de salud (5). 
Las falencias en estos aspectos han sido una constante en Colombia, ya que la configuración de los incentivos en el sistema de salud no está dada para que los aseguradores y prestadores asuman plenamente laresponsabilidad pública que conlleva materializar el goce efectivo del derecho a la salud desde la prestación (6). En consecuencia, el Estado surge como el único actor capaz de reconfigurar las reglas y el sistema de salud, de incidir en el comportamiento de los actores y contrarrestar las fallas del mercado (7), mediante la estipulación de los mecanismos necesarios para garantizar el derecho a la salud y mantener un equilibrio entre los objetivos de bienestar que se quiere lograr a través del sistema de salud y los intereses de los diversos actores que en él participan.

En virtud de ello, el Sistema de Inspección, Vigilancia y Control (IVC) en salud cobra especial relevancia, puesto que se constituye en la herramienta de la que se sirve el Estado para garantizar que los diversos actores del Sistema General de Seguridad Social en Salud (SGSSS) ayuden a alcanzar los objetivos sociales y políticos para los que este se creó. Dicho sistema de IVC está compuesto por el conjunto de actores que conforman la red de controladores del sector, que se encarga del seguimiento a los procesos de $\mathrm{fi}$ nanciación, aseguramiento y prestación de servicios (8).

Desde la perspectiva de la gobernanza y el liderazgo del sistema de salud, el Estado debe adoptar una actitud proactiva, regulando de manera anticipada para prevenir los efectos no deseados, o de manera reactiva frente a los resultados alcanzados para sancionar y reducir al mínimo los efectos negativos. Para que esto sea posible, es necesario contar con sistemas de información en salud (SIS) que garanticen un adecuado seguimiento y monitoreo del SGSSS, desde el cual sea posible definir instrumentos de regulación y mecanismos para hacerlos cumplir $(7,9)$. A su vez, en un círculo virtuoso, la efectividad de dichos instrumentos podrá ser monitoreada a partir de la función de supervisión, promoviendo la transparencia y asegurando que todos los actores del sistema cumplan con su función (5).

En Colombia, los esfuerzos por hacer seguimiento al desempeño del SGSSS encuentran su origen en el Programa de Apoyo a la Reforma en Salud (PARS) 1999-2008, cuyos desarrollos posteriores se tradujeron en avances técnicos y normativos encaminados a mejorar los resultados de la atención en salud $(10,11)$, consolidar el Sistema Integral de Información de la Protección Social (Sispro) (12) y facilitar el flujo de recursos entre los actores del sistema (13).

Sin embargo, no hubo influencia de estos desarrollos en la mejora de la efectividad de la IVC. Por el contrario, estos avances supusieron una pesada carga sobre las funciones de IVC que desarrollaba la Superintendencia, hasta que en el 2007 el Decreto 1018 dotó a la entidad con una estructura acorde con las funciones que le fueron encomendadas en el marco de la Ley 1122 de ese año. La reforma favoreció el desenvolvimiento del organismo y desembocó en la emisión de la Circular nica 047 de 2007. No obstante, el enfoque concentró la mayoría de los esfuerzos de la entidad en responder reclamaciones, sin que las funciones de monitoreo y seguimiento fueran muy claras en ejes diferentes al de $\mathrm{fi}$ nanciamiento (8). Ajustes posteriores buscaron corregir estos problemas y adoptar un enfoque de supervisión basada en riesgos (SBR), a fin de optimizar los esfuerzos del supervisor $(14,15)$.

Sin embargo, en consonancia con el modelo de gestión pública dominante, la IVC ha mantenido su atención en la eficiencia y, por tanto, ha dirigido su atención hacia el riesgo financiero, el riesgo en salud (visto como riesgo financiero), el aseguramiento y la supervisión de cumplimiento en los requisitos de entrada al mercado, por lo que ha resultado poco efectiva para lograr los resultados de salud esperados $(8,16)$, dando lugar a pronunciamientos de la Corte Constitucional por la "violación sistemática del derecho a la salud" $(8,17,18)$.

En este contexto, el propósito de este artículo es evidenciar la capacidad de respuesta que tienen los SIS en Colombia para soportar un modelo de IVC basado en indicadores para la gestión de riesgos y, por tanto, para monitorear y generar alertas tempranas sobre fallas en el desempeño del sistema para la garantía del acceso, el uso y la calidad de los servicios de salud. Los resultados aquí presentados pueden ser útiles para encaminar investigaciones futuras que contribuyan a consolidar conocimientos sobre la SBR y su relación con el derecho a la salud. 


\section{Materiales y método}

La investigación se llevó a cabo mediante un estudio transversal y descriptivo, que se sirvió del enfoque de gestión de riesgos, en el cual la información, los sistemas de monitoreo y las alertas tempranas son centrales (19); de la metodología de análisis de sistemas de información mediante diccionarios y depósitos de datos propuesta por Kendall y Kendall (20); del análisis estructural que, inspirado en el enfoque sistémico (21, 22), permitió la identificación de los componentes (elementos) del "sistema de riesgos para el acceso, uso y calidad” y el análisis en términos de relaciones de influencia/dependencia, dando cabida a la representación de la complejidad en su fase inicial, para posteriormente identificar sus variables principales (puntos críticos de control) mediante el método MIC-MAC.

Adicionalmente, se llevó a cabo un análisis normativo (23) para evaluar y emitir los juicios de valor y establecer las relaciones de influencia/dependencia en el sistema de riesgos, así como la relación entre los derechos que pueden ser afectados al configurarse tales riesgos. Este ejercicio se llevó a cabo para dar cuenta de la situación actual y, a la vez, para identificar las consideraciones hacia el futuro del deber ser, con miras al diseño del sistema de indicadores y la parametrización de las alertas.

El estudio partió de los 281 riesgos derivados de las relaciones de los agentes en el sistema (EPS, IPS, entes territoriales, Fosyga, Superintendencia Nacional de Salud (SNS), Ministerio de Salud) (24). A partir de allí, se condujo una revisión del marco normativo y regulatorio vigente para la generación y el reporte de información asociada a los riesgos. Se revisaron 116 normas (24 leyes, 29 decretos, 52 resoluciones, 2 acuerdos, 8 circulares y un concepto) conexas con 131 riesgos asociados a la operación territorial del SGSSS.

Asimismo, se realizó una caracterización de los sistemas de información en salud, a partir de un conjunto de atributos $^{2}$ estructurados en un modelo de entidad-relación, y en el interior de cada sistema se identificaron los datos e indicadores relacionados con el acceso, el uso y la calidad. Las fuentes fueron: Sistema de información de la Circular 047 del 2007 de la SNS; Sistema de Peticiones, Quejas, Reclamos y Denuncias PQRD de la SNS; Sistema de Vigilancia de Salud Pública (Sivigila); Registro individual de prestaciones en salud (RIPS); Sistema Obligatorio de Garantía de la Calidad (SOGC); Registro Especial de Prestadores de Servicios de en Salud (REPS); Sistema de Gestión de Hospitales Públicos (SIHO); Registronico de Afiliados (RUAF).

Luego de varias etapas de criba, se toman 131 riesgos relacionados con las competencias de las direcciones territoriales de salud (DTS). Esto, porque gran parte de las dificultades para el ejercicio de la IVC se derivan del alto grado de centralización de la supervisión en las instancias del orden nacional (25). Estos riesgos fueron clasificados en ocho categorías operativas o núcleos, de acuerdo con su interdependencia en la cadena de prestación de los servicios de salud y con base en los preceptos del marco normativo e institucional de operación del SGSSS a saber: afiliación y representación del afliado, redes de prestación de servicios de salud, habilitación y auditoría a IPS, sostenibilidad fnanciera de la IPS, control socialy protección al usuario, seguridad del paciente, trato digno y rutas integrales de atención.

La siguiente fase consistió en vincular los indicadores, teniendo en cuenta dos cuestionamientos: ¿el indicador proporciona información para la IVC del riesgo al que está siendo vinculado? y ¿el indicador cumple con los criterios $S M A R T^{4}$ ? En total se revisaron 403 indicadores y se clasificaron de acuerdo con su relación con el riesgo, en directo, indirecto y propuesto para ser construido de fuente primaria.

Posteriormente, se consolidó una base de datos en la que se asociaron riesgos, indicadores, atributos del derecho fundamental a la salud y los principios expuestos en la Ley Estatuaria de Salud (26), lo que permitió priorizar los riesgos y sus indicadores en cuanto fuesen una expresión observable de las afectaciones a los derechos de acceso, uso y calidad de los servicios de salud.

Con este insumo se construyó una batería con 102 indicadores, que se sometió a un proceso de validación por parte de funcionarios de las DTS del departamento de Cundinamarca, la Secretaría de Salud de Bogotá y de los municipios de Tocancipá, Cajicá, Zipaquirá y Ubaté (15 participantes). 
A fin de establecer los riesgos clave y definir los puntos críticos de control (PCC) (19), se llevó a cabo un análisis de impacto cruzado en el software MIC-MAC. De acuerdo con el procedimiento descrito por Martínez (27), cuatro investigadores calificaron la influencia de cada variable (riesgo) en relación con las demás, a partir de una escala de probabilidad de influencia: $0=$ no influye, $1=$ influencia débil, $2=$ influencia media, 3 = influencia fuerte y $\mathrm{P}=$ influencia probable. La calificación final se estimó promediando los puntajes dados a cada relación. Se trazaron mapas de impacto directo/indirecto a partir de los parámetros predefinidos en el software de análisis. Así, la hipótesis teórica que se plantea es que la intervención temprana sobre ese conjunto de riesgos críticos, como base mínima, contribuirá a un mejor desempeño del sistema de salud. La figura 1 sintetiza el enfoque metodológico empleado.

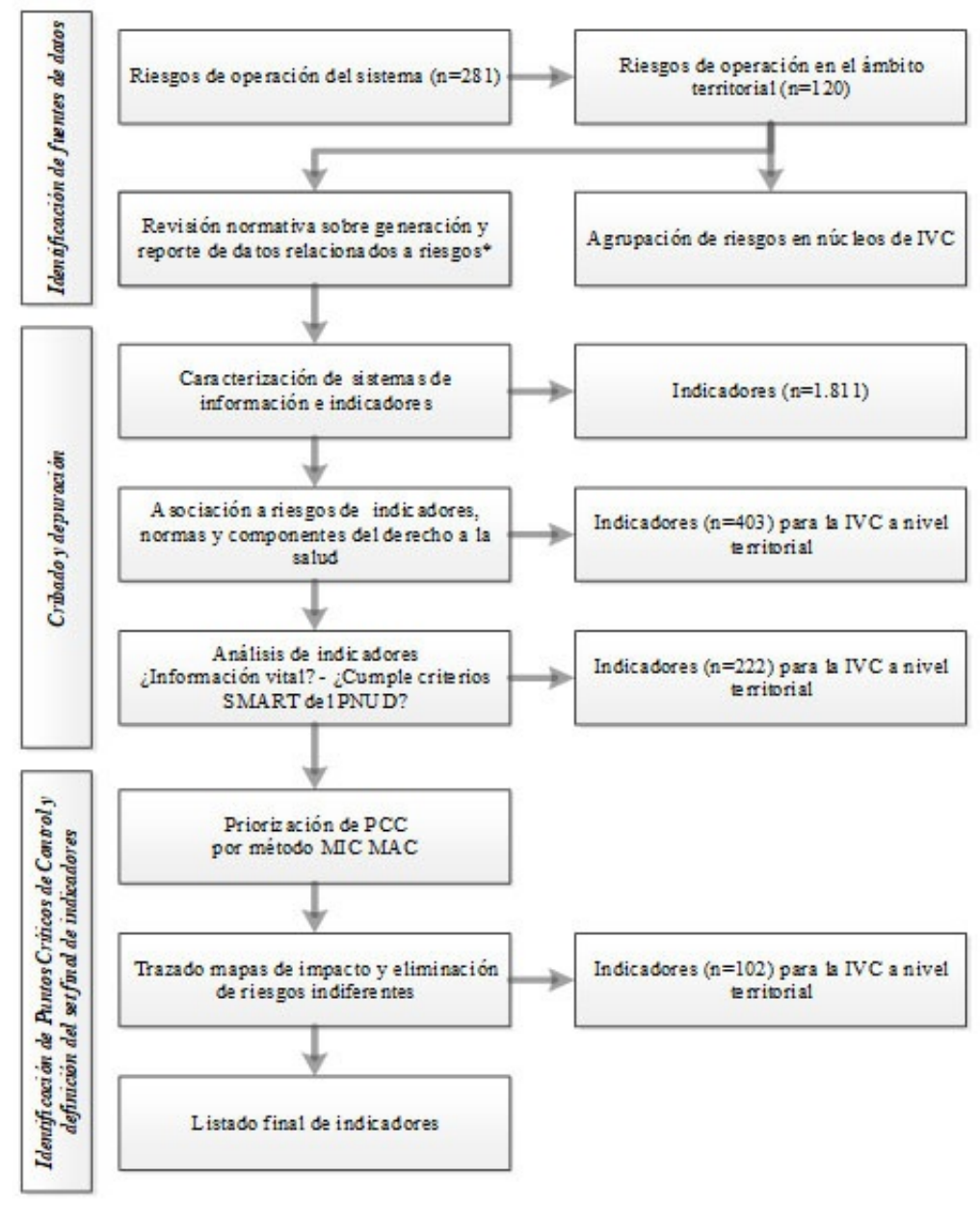

*PCC: Puntos Criticos de Control

FIGURA 1

Proceso de revisión de SIS para la IVC

Fuente: elaboración propia. 


\section{Resultados}

\section{Los núcleos de riesgo y los componentes del derecho a la salud}

De los 131 riesgos susceptibles de ser monitoreados desde los niveles territoriales, el primer lugar correspondió al núcleo de afiliación y representación del afiliado, con una participación del $29 \%$. Una proporción que prácticamente duplica al siguiente núcleo, de seguridad del paciente (16\%). El tercer lugar lo ocupó la habilitación y auditoría de IPS (12\%), seguida de las rutas integrales de atención (11\%), la sostenibilidad financiera de las IPS, que junto con el control social y la participación del usuario tuvieron similar participación (9\%). Finalmente, el trato digno y las redes integrales de atención en salud tuvieron la participación más baja (con el $8 \%$ y el $7 \%$, respectivamente).

Con el proceso de priorización y definición de los PCC se obtuvieron 51 riesgos que, al relacionarse con los componentes del derecho fundamental a la salud, (26), muestran interacciones múltiples entre ellos, aunque ciertos núcleos de riesgo resultan ser dominantes a la hora de afectar una dimensión del derecho (figura 2). Por esta razón, la SBR debe comprenderse como un ejercicio sistémico de seguimiento, prevención y mitigación de todas aquellas situaciones que comprometen la garantía del derecho a la salud y, por tanto, involucra los distintos niveles territoriales según las competencias a nivel centralizado y descentralizado, haciendo efectivo el principio de subsidiariedad del Estado.

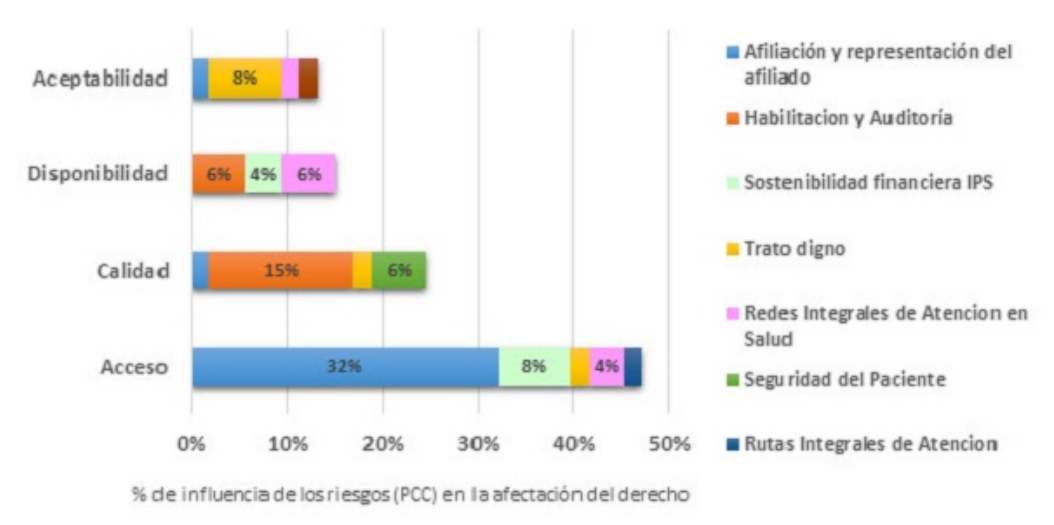

FIGURA 2

Distribución de los riesgos - PCC por núcleo de IVC en los componentes del derecho a la salud. Fuente: elaboración propia.

Sobre la dimensión del acceso, predominan los riesgos asociados a la afiliación y representación del afiliado, específicamente los relacionados con el proceso de autorización/negación de servicios, procedimientos o insumos especializados, los tiempos de espera para ello (oportunidad) y la actualización en tiempo real de registros que acreditan el derecho de afiliado. En menor medida, dos núcleos que involucran al sistema de prestación de servicios y su relación con las EPS: la sostenibilidad financiera de las IPS y las redes integrales de atención en salud. Estos factores implican las capacidades de la SNS sobre las EPS para prevenir, vigilar o sancionar frente a las infracciones en su rol de representación del afiliado y del pago oportuno a sus redes de servicios.

La dimensión de la calidad se vio menoscabada principalmente por el núcleo de habilitación y auditoria, seguido de la seguridad del paciente. Tales riesgos se refieren principalmente al incumplimiento de los estándares de habilitación por parte de las IPS, a la inadecuada gestión clínica de la enfermedad y de los eventos adversos. En general, estos riesgos son el resultado de la materialización de situaciones que superan el proceso de prestación de los servicios de salud derivados de las capacidades y competencias de IVC que tienen 
las DTS. Estos factores también determinan las características de la oferta en los contextos territoriales y, en consecuencia, la forma en que se estructura la prestación dentro de la red de cada asegurador (28).

Por su parte, la dimensión de disponibilidad es afectada en igual medida por los riesgos asociados a la habilitación y auditoría de IPS y aquellos vinculados con la conformación y funcionalidad de las redes para la prestación de servicios de salud por parte de las EPS, que expresan situaciones de restricción o imposibilidad de ofrecer un portafolio completo de servicios de forma integral, especialmente en municipios y departamentos de menor categoría. El siguiente lugar lo ocupa el núcleo de la sostenibilidad financiera de las IPS.

Por último, la dimensión de la aceptabilidad está comprometida principalmente por los riesgos propios del trato digno, en particular, la acogida por parte del personal administrativo y de salud, las fallas de información sobre procedimientos y atención, las faltas a la ética médica y la no existencia de un enfoque diferencial en los casos requeridos por la ley, entre otros. Existen otros riesgos que se asocian a núcleos ya descritos y, aunque su incidencia sobre esta dimensión es mucho menor, permiten confirmar los supuestos sobre la interdependencia y perspectiva sistémica de los riesgos y de las dimensiones del derecho.

\section{Los puntos críticos de control para la IVC basada en riesgos a nivel territorial}

Como puede observarse en la figura 3, la priorización de los riesgos basada en las relaciones de causalidad/ consecuencia sitúan al núcleo de afiliación y representación del afiliado como aquel con el mayor número de riesgos críticos en el cuadrante "activos como determinantes". Si se tiene en cuenta que el Decreto 2353 de 2015 se expidió con el objeto de mitigar las dificultades derivadas del proceso de afiliación, el monitoreo futuro debería evidenciar la disminución progresiva en la frecuencia de estos riesgos. Por su parte, en la zona de "reguladores del sistema" (zona central) se ubicaron situaciones asociadas a los trámites burocráticos (autorizaciones en la EPS) para el uso de los servicios de salud, lo que indicaría la necesidad de generar mecanismos regulatorios (incentivos y sanciones) tendientes a reducir la incidencia de circunstancias que afecten el acceso y la aceptabilidad de los servicios de salud.

En el sector de los "determinantes activos" se ubicaron los riesgos relacionados con la habilitación y auditoría de IPS, seguidos por la sostenibilidad financiera de los prestadores. En este caso, la configuración de los riesgos afecta la prestación de los servicios de atención en salud y evidencia la falta de efectividad y el incumplimiento de las EPS y las direcciones territoriales de salud en la vigilancia de la red de prestadores, así como del nivel nacional en la supervisión de la regulación de la oferta y los contratos que median las relaciones entre aseguradores, prestadores y pagadores en el sistema.

Finalmente, se encuentran los núcleos de trato digno y seguridad del paciente, los cuales expresan principalmente las relaciones entre las IPS y los usuarios, con una dependencia directa de los riesgos precedentes que amenazan las dimensiones de calidad y aceptabilidad del derecho a la salud. 


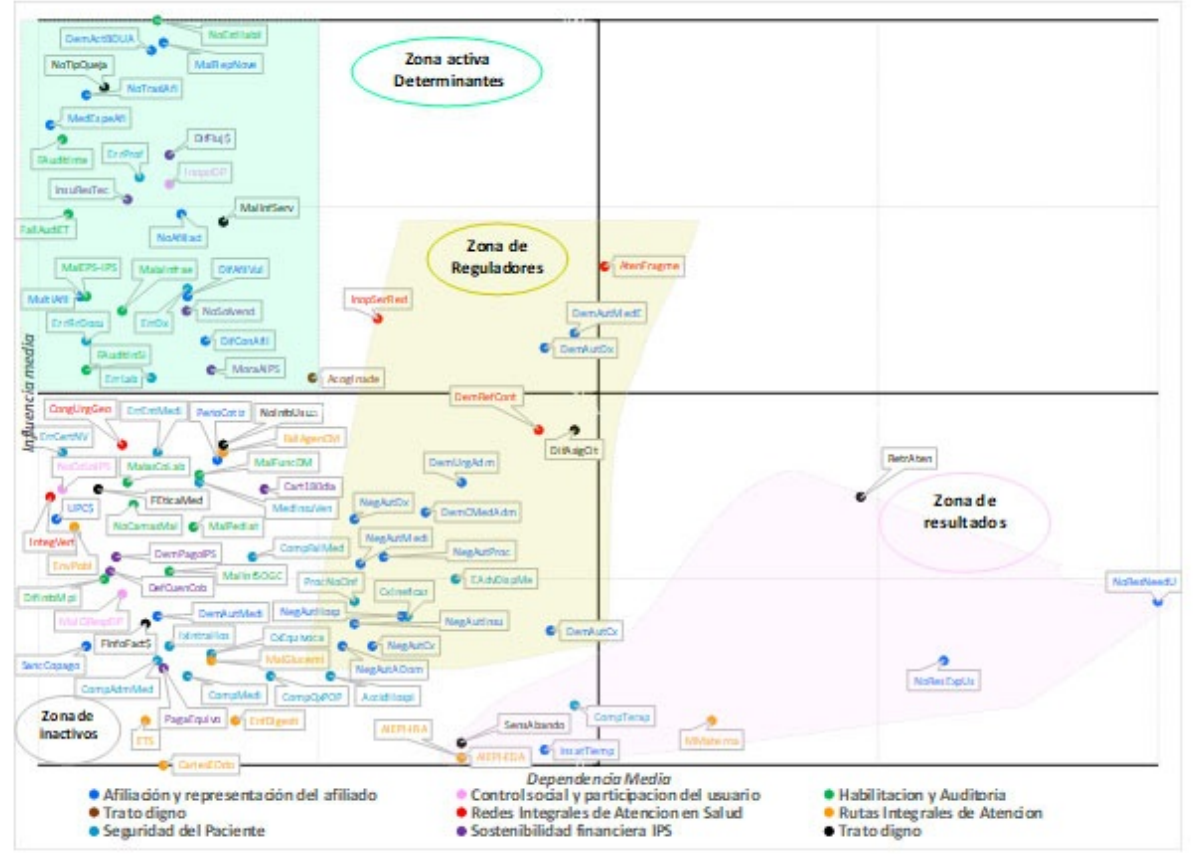

FIGURA 3

Matriz de impactos cruzados para la priorización de riesgos y sus indicadores. Fuente: elaboración propia con base en Eslava, Camelo, Mejía y Mina (29)

\section{La capacidad de respuesta de los sistemas de información para la supervisión basada en riesgos (SBR)}

Pese a que el marco normativo de la IVC es extenso, para el conjunto de riesgos definidos en el sistema de seguimiento y monitoreo predomina la regulación administrativa enfocada en los riesgos intraorganizacionales y, en menor medida, en aquellos componentes que expresan la garantía del derecho a la salud.

Bajo el entendido de que la SBR es un proceso sistemático de seguimiento a los puntos donde pueden presentarse fallas, es claro que requiere sistemas de información con indicadores y sistemas de alerta para orientar la toma de decisiones desde una perspectiva preventiva. Sin embargo, el análisis de la disponibilidad de los datos y de la regulación muestra serias falencias en el seguimiento de los riesgos definidos en este estudio.

\section{La producción y los flujos de información en el sistema}

Desde la regulación existente, la Circular Única 047 de 2007 de la SNS representa la piedra angular para el ejercicio de la IVC en el SGSSS, ya que define los instrumentos, las reglas y los atributos para el reporte de indicadores. En los anexos técnicos analizados (EPS régimen contributivo y subsidiado, IPS pública y privada y entidades territoriales de salud), se evidencia que la mayor parte de los archivos (62,2\%) se concentra en hacer seguimiento a la sostenibilidad financiera de los actores, el 22,2\% a los contratos para la conformación de redes y el restante $15,6 \%$ en el seguimiento a las actividades de IVC de las DTS (indicadores de tecnología biomédica, seguimiento al régimen subsidiado y ejecución de las actividades del Plan de Intervenciones Colectivas [PIC]).

Por otra parte, el Sistema de Registros, Observatorios y Sistemas de Seguimiento (ROSS), que se encuentra en proceso de integración al Sispro, contaba en el año 2013 con un total de 16.412 indicadores adicionales 
a los 1811 que ya se generaban (30). Si bien el ROSS dispone de un volumen considerable de indicadores y constituye una herramienta valiosa para el seguimiento de la situación de la salud a escala nacional, no cuenta con una utilidad práctica para supervisar el desempeño del sistema desde los entes territoriales.

A partir de la citada revisión por parte del Ministerio de Salud en el año 2014, y retomada por esta investigación, se seleccionaron 69 indicadores compatibles con algunos de los riesgos por monitorear. Además, se propuso la creación de 33 nuevos indicadores, debido a que los existentes no dan cuenta de todos los riesgos dentro de la IVC. La información para el cálculo de estos indicadores propuestos la generan actualmente las entidades territoriales y las EPS o IPS, aunque de forma local, aislada y no siempre automatizada.

No obstante, al recabar la información de los 69 indicadores existentes, solo se logró la recolección de información correspondiente a 15 de ellos, debido a tres razones: restricción en el acceso a los datos, falta de integración entre los sistemas de información existentes y falta de claridad por parte de las mismas entidades generadoras de la información sobre los datos que tienen en sus bodegas de datos.

Por otra parte, el panorama para la recolección de la información de los 33 indicadores propuestos no fue más alentador, pues no existen reglas de estandarización para su producción, almacenamiento y uso. A pesar de que los actores del sistema reconocen el potencial que esta información les puede aportar en sus procesos de IVC, se carece de personal suficiente para generar el dato en el corto plazo.

Aunque en algunos casos se identificó más de un indicador para un mismo riesgo, en la práctica fueron pocos los indicadores que se calificaron como "directos" para dar cuenta de los riesgos bajo estudio. A ello se suma que, si bien de acuerdo con las fuentes consultadas el indicador existe y se encuentra regulado, el acceso a esta información está centralizado en la SNS, no está estructurada en un sistema de información o su acceso es de carácter restringido para el público, pues prima el derecho de la entidad privada a la salvaguarda de su información.

De esta forma, los dos grandes pilares de la recolección, compilación, análisis, síntesis y comunicación de la información sanitaria para la toma de decisiones en el SGSSS se presentan como bodegas de datos aisladas, sin mecanismos que faciliten su interoperabilidad y funcionalidad para el seguimiento a los procesos y resultados para la garantía del acceso, uso y calidad de los servicios de salud. Esta brecha se hizo evidente en la medida en que aun cuando algunos riesgos estuvieron regulados normativamente, no existían mecanismos e información suficiente para verificar su cumplimiento.

Debido a la cantidad de vigilados (EPS, IPS, DTS) y actores con competencias en IVC (MSPS, SNS, DTS, Instituto Nacional de Salud, Invima, procuradurías, personerías y defensorías), el flujo de información en el sector salud es muy complejo. Adicionalmente, existen varios niveles para su agregación de acuerdo con la entidad a la que se reporta, por lo que las duplicidades, las bajas coberturas y la poca calidad en los datos son comunes. Estas condiciones, sumadas al desconocimiento de algunos actores sobre la existencia de registros estandarizados en el sistema, se traduce en el poco uso de la información recabada en las bodegas del Sispro o en la SNS.

La fuente original de información en el sistema se encuentra en las unidades primarias generadoras del dato (UPGD), que pueden ser IPS, prestadores independientes o laboratorios, que reportan lo referente a prestaciones, vacunación (PAI), capacidad instalada, enfermedades de interés en salud pública, cartera de aseguradores y sus propias inversiones. En el siguiente nivel se encuentran los aseguradores, quienes en general consolidan la información de los prestadores en su red y reportan al Gobierno central. En algunos casos (enfermedades de interés), las UPGD reportan directamente a la DTS municipal, la cual extiende la información al nivel departamental, y este a la nación.

Los organismos de nivel nacional se encargan de validar y consolidar la información reportada por sus vigilados y tomar las medidas pertinentes frente a desviaciones en el comportamiento esperado. Sin embargo, no se encontraron mecanismos para evaluar los datos en los niveles más bajos de la pirámide, lo que supone un aumento importante en el tiempo de respuesta frente a situaciones anómalas en los niveles más próximos a los 
ciudadanos, lo que explica el escaso reconocimiento social de la IVC entre los usuarios del sistema. La figura 4 presenta el esquema general del reporte de información para la IVC en el sistema; no se incluye a los demás miembros de la red de controladores, pues la información que se les reporta se hace por solicitud directa.

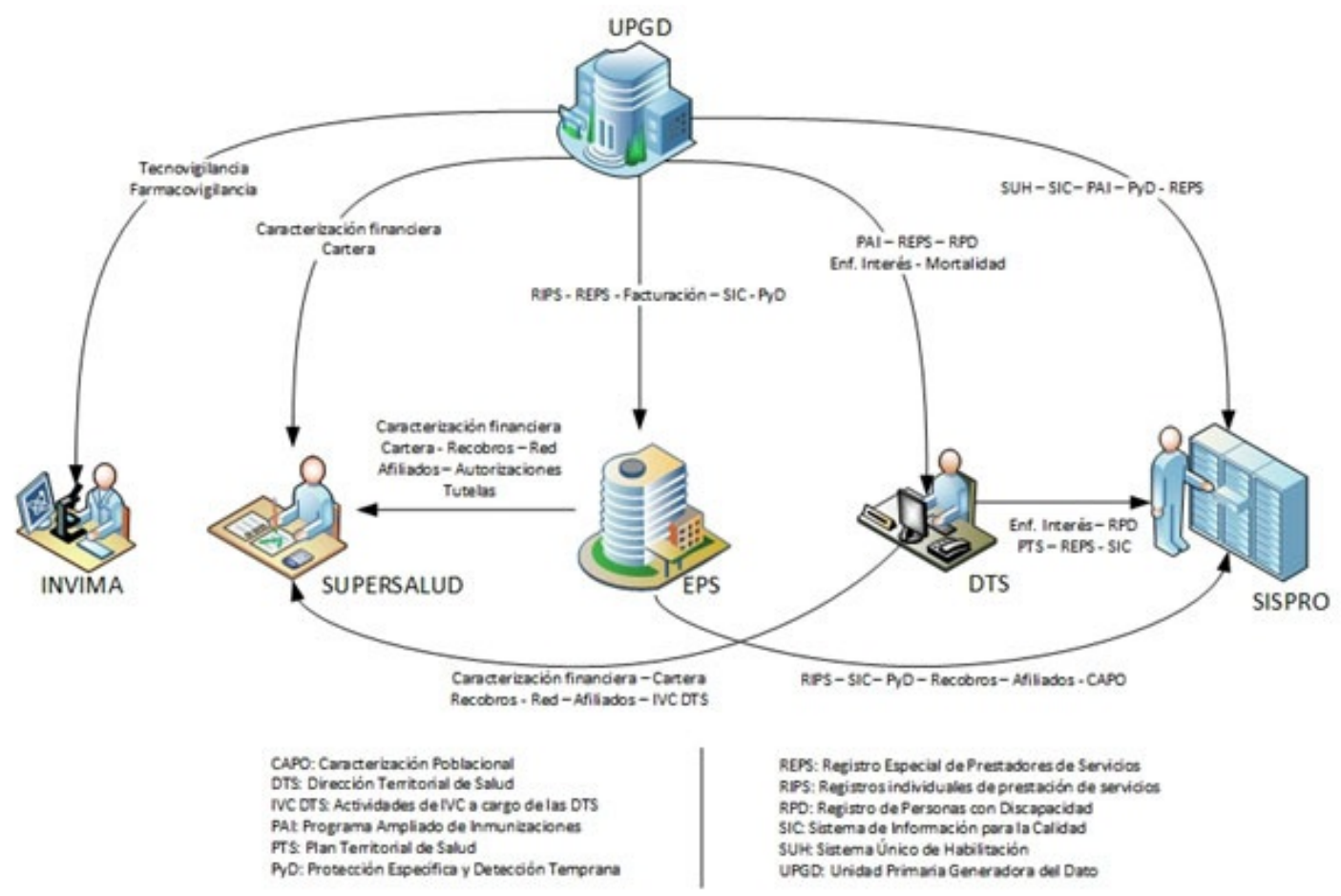

FIGURA 4

Esquema general para el reporte de información para la IVC en el SGSS

Fuente: elaboración propia.

No se encontraron mecanismos de retroalimentación con respecto a la calidad de la información y errores en el registro y tampoco se definen baremos para el análisis de la información. Estas condiciones no solo dificultan el procesamiento de datos en las instancias de IVC, sino que resultan en la inexistencia de mecanismos objetivos de sanción frente al reporte de anomalías por parte de los actores.

\section{Los indicadores para la IVC basada en el derecho}

Si se tiene en cuenta el carácter sistémico de la IVC como un mecanismo para la garantía del derecho, se decidió que deberían tomarse indicadores de todas las fuentes para ligarlos a los riesgos. Con el objeto de decantar la información más relevante, el equipo investigador desarrolló un análisis de impactos cruzados (MIC MAC) en el que se determinó que los mejores resultados podrían obtenerse con el seguimiento de 71 riesgos críticos que comprendían 102 indicadores (anexo 1).

El núcleo que concentró más riesgos - e indicadores- fue afiliación y representación del afiliado, con 40 indicadores, seguido de habilitación y auditoria $(\mathrm{n}=16)$, sostenibilidad financiera IPS $(\mathrm{n}=13)$, trato digno $(\mathrm{n}=11)$, redes integrales de atención en salud $(\mathrm{n}=10)$, rutas integrales de atención en salud $(\mathrm{n}=5)$, seguridad del paciente $(\mathrm{n}=4)$ y control social y participación del usuario $(\mathrm{n}=3)$.

Este set de indicadores constituye una herramienta para hacer seguimiento a la garantía del derecho, pero no se considera un fin en sí mismo, sino un medio a través del cual la institucionalidad provee las condiciones para el acceso y el uso de los servicios de salud a la luz de una calidad técnica —efectividad clínica一, certificada por el cumplimiento de las condiciones de habilitación y del seguimiento a la prestación para mejorar la situación de salud de los colombianos. 


\section{Discusión}

El entramado normativo que regula las acciones de los actores en el SGSSS no logra reconciliar los intereses particulares y los colectivos, en aras del bien común y la protección de los derechos de los usuarios. Si bien a partir de la emisión de la Sentencia T-760 la Corte Constitucional elevó la salud a la categoría de derecho fundamental, la realidad operativa del sistema muestra que las lógicas económicas siguen prevaleciendo frente al derecho (31), dejando por fuera la esencia de lo que debería ser un sistema en salud, esto es, una herramienta del Estado para garantizar a sus ciudadanos el goce efectivo del derecho (GED), expresado en el acceso, el uso y la calidad de los servicios; en la promoción de la salud individual y colectiva; en la adopción de un enfoque de riesgos que se sitúe en una lógica preventiva, pero que también conciba el riesgo en salud como dispositivo de alerta para movilizar la gestión integral de la atención.

Aunque los ajustes más recientes al sistema se han orientado a proteger y garantizar el derecho, no se cuenta con instrumentos que permitan monitorear con agilidad la forma en que los actores institucionales del sistema desempeñan su función. Si bien la norma resulta garantista en muchos sentidos, la incapacidad de los actores institucionales del sistema para verificar su cumplimiento permite que se presenten desviaciones en su comportamiento. $\mathrm{Y}$ aun cuando los organismos de control gubernamentales se esfuerzan por limitar el riesgo de incumplimiento, las dinámicas del mercado evolucionan más rápido que los esfuerzos regulatorios del supervisor $(14,15)$.

En este sentido, los autores concuerdan con Giuffrida, Pinzón y Piñeros en que los recursos de los organismos con responsabilidades de IVC, al ser limitados, deben usarse de la manera más eficiente posible. Esto, a partir de un enfoque de SBR, donde el rol del supervisor deja de ser el de verificar el cumplimiento normativo, y se encamina a evaluar si los controles destinados a evitar la materialización de los riesgos son adecuados (32). Y, adicionalmente, es una función dinámica y flexible a las condiciones del mercado (15).

Sin embargo, la implementación de un sistema de SBR debe contar con un sistema de información que soporte de manera ágil y oportuna el flujo de datos que emana desde los vigilados. Así, los autores toman la definición de la OMS citada por Ovies y Agudelo (33) de un SIS como un conjunto organizado y armónico de normas, personas, procesos, hardware y software, diseñados con el fin de producir información relevante para el sector y proveer las bases para la toma de decisiones a partir de cuatro funciones: (1) generación; (2) compilación; (3) análisis-síntesis, y (4) comunicación y uso de la información sanitaria (34).

Si se considera lo anterior, resulta evidente que en Colombia no se cuenta con un sistema de información para el ejercicio de la IVC. Esto ocurre porque los datos necesarios para el seguimiento integral al desempeño de los actores, a la luz de la protección del derecho, se encuentran en el mejor de los casos separados, debido a que el desarrollo de los SIS se ha dado de manera fragmentada en nuestro país. Incluso, muchas veces, ese seguimiento ha sido resultado de esfuerzos individuales $(34,35,36)$, desconociendo el principio de cooperación que debe primar entre los organismos del Estado.

Como consecuencia, es posible encontrar diferentes niveles de desarrollo, tanto en los sistemas de información como en su integración a la bodega de datos del Sispro. En general, aunque los desarrollos en la plataforma y en el registro de información han sido positivos, muchas de las fuentes relacionadas en el ROSS aún no se encuentran disponibles para su consulta. Adicionalmente, se pudieron constatar los hallazgos de Bernal-Acevedo y Forero-Camacho y León (36), en torno a lo incipiente de los avances en materia de interoperabilidad técnica, semántica, de infraestructura, organizacional y legal, lo que redunda en dificultades para la recolección, almacenamiento, análisis y reporte de datos por parte de los actores territoriales del sistema, que sumados a la inviabilidad técnica que representa la validación de registros individuales a nivel central, se traduce en serios problemas de calidad dentro de las fuentes.

Muchos de los registros que se usan para hacer seguimiento a las condiciones epidemiológicas de la población (morbimortalidad, brotes, situación de salud, discapacidad y bienestar), a los inputs del SGSSS, y sus procesos relacionados (política, organización, infraestructura, instalaciones y equipo; costos, recursos 
humanos y financieros) no son utilizados para determinar el desempeño del sistema (accesibilidad, uso y calidad, de los servicios, la capacidad de respuesta ante las necesidades del usuario y la protección frente al riesgo financiero que conlleva la enfermedad) (34). En general, la recolección de los datos favorece el interés financiero sobre el desenvolvimiento del sistema, y se relegan a un segundo plano condiciones que se han convertido en aspectos críticos desde la percepción de los usuarios.

Por otra parte, la falta de retroalimentación hacia los generadores de información y los largos tiempos para el procesamiento, análisis y comunicación de los datos reportados por los agentes del sistema (8) han llevado a una pérdida del reconocimiento de las funciones de IVC, tanto en el interior del sistema como por parte de los usuarios (25). Este hecho resulta paradójico si consideramos la multiplicidad de fuentes normativas que prescriben y regulan la producción de información sobre el SGSSS y en particular para los riesgos objeto de estudio, pues todos sin excepción estaban contenidos en más de un instrumento (leyes, decretos, resoluciones y circulares). Quizá el gran vacío está en los inadecuados mecanismos de regulación para que las entidades receptoras hagan una gestión efectiva de dicha información y puedan proceder con los mecanismos de sanción adecuados ante el incumplimiento en oportunidad y estándares de reporte. Sumado a ello, la falta de baremos para la medición objetiva de desviaciones en el comportamiento de los actores también ha redundado en dificultades a la hora de aplicar sanciones ante el reporte de condiciones que afectan el libre ejercicio del derecho.

En conclusión, se encontró que si bien la información que producen los actores del sistema de salud es pertinente y suficiente para efectuar el seguimiento al acceso, el uso y la calidad de los servicios que se prestan en el marco del SGSSS, esta se encuentra dispersa en diversas fuentes, que presentan problemas de interoperabilidad técnica, semántica y de infraestructura, lo que dificulta contrastar la información administrativa con los resultados de la atención en salud que reposan en el Sispro.

\section{Agradecimientos}

Artículo de investigación asociado al proyecto Desarrollo de un Sistema de Monitoreo, de intervenciones regulatorias y de inspección, vigilancia y control en el Sistema General de Seguridad Social en Salud(2014-2017). El proyecto fue financiado por Colciencias y la Pontificia Universidad Javeriana, en el marco del programa Desarrollo y evaluación de un modelo integrado para la inspección, vigilancia y control del acceso oportuno, el uso adecuado y la calidad en la prestación de servicios en el SGSSS colombiano, convocatoria 576-2012. 


\section{Anexo 1. Especificación de los riesgos por núcleo}

\begin{tabular}{|c|c|c|}
\hline Nombre corto & Nombre Largo & Núcleo \\
\hline AcogInade & Acogida inadecuada por parte del personal. & Trato Digno \\
\hline Cart180dia & Incremento de la cartera mayor a 180 días. & $\begin{array}{l}\text { Sostenibilidad financiera de } \\
\text { la IPS }\end{array}$ \\
\hline DefCuenCob & Deficiencias en la organización de cuentas por cobrar. & $\begin{array}{l}\text { Sostenibilidad financiera de } \\
\text { la IPS }\end{array}$ \\
\hline DefCuenCob & Deficiencias en la organización de cuentas por cobrar. & $\begin{array}{l}\text { Sostenibilidad financiera de } \\
\text { la IPS }\end{array}$ \\
\hline DemActBDUA & $\begin{array}{l}\text { Demora en la actualización de las bases de datos de los } \\
\text { usuarios. }\end{array}$ & $\begin{array}{l}\text { Afiliación y representación } \\
\text { del Afiliado }\end{array}$ \\
\hline DemAutDx & $\begin{array}{l}\text { Demoras de la autorización de exámenes de laboratorio o } \\
\text { diagnósticos. }\end{array}$ & $\begin{array}{l}\text { Afiliación y representación } \\
\text { del Afiliado }\end{array}$ \\
\hline DemAutMedE & Demoras de la autorización de consultas médicas especializadas. & $\begin{array}{l}\text { Afiliación y representación } \\
\text { del Afiliado }\end{array}$ \\
\hline DemCMedAdm & $\begin{array}{l}\text { Estancias prolongadas de usuarios en el servicio de citas } \\
\text { médicas por dificultades administrativas. }\end{array}$ & $\begin{array}{l}\text { Afiliación y representación } \\
\text { del Afiliado }\end{array}$ \\
\hline DemRefCont & Demoras en los procesos de referencia/contra referencia & $\begin{array}{l}\text { Afiliación y representación } \\
\text { del Afiliado }\end{array}$ \\
\hline DemUrgAdm & $\begin{array}{l}\text { Estancias prolongadas de usuarios en el servicio de urgencias } \\
\text { por dificultades administrativas. }\end{array}$ & $\begin{array}{l}\text { Afiliación y representación } \\
\text { del Afiliado }\end{array}$ \\
\hline DifAfilVul & $\begin{array}{l}\text { Dificultades para ubicar población pobre no afiliada y } \\
\text { desplazados. (difícil localización de beneficiarios para su } \\
\text { afiliación) }\end{array}$ & $\begin{array}{l}\text { Afiliación y representación } \\
\text { del Afiliado }\end{array}$ \\
\hline DifAsigCit & $\begin{array}{l}\text { Dificultades del usuario para la comunicación con las líneas de } \\
\text { atención para la asignación de citas. }\end{array}$ & Trato Digno \\
\hline DifConAfil & $\begin{array}{l}\text { Dificultades en la continuidad de la afiliación ante retiro de } \\
\text { EPS-S debido a trabas para autorizar la cesión de contratos. }\end{array}$ & $\begin{array}{l}\text { Afiliación y representación } \\
\text { del Afiliado }\end{array}$ \\
\hline DifFluj\$ & Dificultades en el flujo de recursos. & $\begin{array}{l}\text { Sostenibilidad financiera de } \\
\text { la IPS }\end{array}$ \\
\hline ErrDx & Fallas diagnósticas. & Seguridad del Paciente \\
\hline ErrFirDocu & $\begin{array}{l}\text { Error al firmar y no revisar los resultados de exámenes o } \\
\text { pruebas diagnósticas realizados por otro profesional. }\end{array}$ & Seguridad del Paciente \\
\hline ErrProf & $\begin{array}{l}\text { Error del profesional al no seguir las indicaciones y/o solicitud } \\
\text { de la referencia emitida. }\end{array}$ & Seguridad del Paciente \\
\hline FallAudiET & $\begin{array}{l}\text { Baja calidad en los servicios prestados a la población elegible no } \\
\text { afiliada ante fallas en la auditoría por parte de la entidad } \\
\text { territorial. }\end{array}$ & Habilitación y Auditoría \\
\hline FAuditInSi & Falta de auditorías concurrentes. (in situ) & Habilitación y Auditoría \\
\hline FAuditInte & Fallas en la aplicación de auditorías internas. & Habilitación y Auditoría \\
\hline FEticaMed & Fallas en la ética médica. & Trato Digno \\
\hline InopoDP & $\begin{array}{l}\text { Inoportunidad en la respuesta a las solicitudes, derechos de } \\
\text { petición o requerimientos efectuados por los usuarios. }\end{array}$ & $\begin{array}{l}\text { Control Social y } \\
\text { Participación del Usuario }\end{array}$ \\
\hline InsuResTec & Insuficiencia de reservas técnicas. & $\begin{array}{l}\text { Sostenibilidad financiera de } \\
\text { la IPS }\end{array}$ \\
\hline MalaInfrae & $\begin{array}{l}\text { Infraestructura deficiente de salas de cirugía, camillas, etc., o } \\
\text { dotación inapropiada. }\end{array}$ & Habilitación y Auditoría \\
\hline MalasCoLab & Laboratorios en malas condiciones. & Habilitación y Auditoría \\
\hline MalEPS-IPS & $\begin{array}{l}\text { Fallas en la asesoría a las EPS e IPS al adelantar los procesos de } \\
\text { auditoría tendientes a mejorar la calidad de la atención en } \\
\text { salud. }\end{array}$ & Habilitación y Auditoría \\
\hline
\end{tabular}




\begin{tabular}{|c|c|c|}
\hline Nombre corto & Nombre Largo & Núcleo \\
\hline MalFuncDM & Funcionamiento defectuoso de dispositivos médicos. & Habilitación y Auditoría \\
\hline MalInfServ & $\begin{array}{l}\text { Mala o deficiente información sobre la prestación de los } \\
\text { servicios. }\end{array}$ & Trato Digno \\
\hline MalInfSOGC & $\begin{array}{l}\text { Fallas en el contenido, calidad y reporte de la información que } \\
\text { conforma el Sistema de Información para la Calidad. }\end{array}$ & Habilitación y Auditoría \\
\hline MalPediat & Condiciones inadecuadas de salas de neonatos y pediatría. & Habilitación y Auditoría \\
\hline MalRepNove & Inconsistencias en los reportes de las novedades & $\begin{array}{l}\text { Afiliación y representación } \\
\text { del Afiliado }\end{array}$ \\
\hline MedEspeAfi & $\begin{array}{l}\text { Medidas especiales por parte de organismos de control que } \\
\text { afecten el proceso de afiliación. }\end{array}$ & $\begin{array}{l}\text { Afiliación y representación } \\
\text { del Afiliado }\end{array}$ \\
\hline MoraAIPS & $\begin{array}{l}\text { Incurrir en sobrecostos financieros asociados a demoras en el } \\
\text { pago a las IPS. }\end{array}$ & $\begin{array}{l}\text { Sostenibilidad financiera de } \\
\text { la IPS }\end{array}$ \\
\hline MultiAfil & Incurrir en multiafiliación. & $\begin{array}{l}\text { Afiliación y representación } \\
\text { del Afiliado }\end{array}$ \\
\hline NegAutDx & $\begin{array}{l}\text { Negación de la autorización para exámenes diagnósticos o de } \\
\text { laboratorio. }\end{array}$ & $\begin{array}{l}\text { Afiliación y representación } \\
\text { del Afiliado }\end{array}$ \\
\hline NegAutMedi & Negación de la autorización de medicamentos & $\begin{array}{l}\text { Afiliación y representación } \\
\text { del Afiliado }\end{array}$ \\
\hline NegAutProc & Negación de la autorización de procedimientos POS. & $\begin{array}{l}\text { Afiliación y representación } \\
\text { del Afiliado }\end{array}$ \\
\hline NoAfiliaci & Negación de la afiliación. & $\begin{array}{l}\text { Afiliación y representación } \\
\text { del Afiliado }\end{array}$ \\
\hline NoCamasMal & Falta de disponibilidad de camas o en deficientes condiciones. & Habilitación y Auditoría \\
\hline NoCoSoIPS & $\begin{array}{l}\text { Incumplir el ejercicio de control social, al no lograr la } \\
\text { participación activa de la comunidad en los procesos de la IPS. }\end{array}$ & $\begin{array}{l}\text { Control Social y } \\
\text { Participación del Usuario }\end{array}$ \\
\hline NoEstHabil & Incumplimiento de estándares de habilitación. & Habilitación y Auditoría \\
\hline NoSolvenci & $\begin{array}{l}\text { Incumplimiento de margen de solvencia (iliquidez) para } \\
\text { cumplir obligaciones con terceros (proveedores de bienes, IPS o } \\
\text { usuarios). }\end{array}$ & $\begin{array}{l}\text { Sostenibilidad financiera de } \\
\text { la IPS }\end{array}$ \\
\hline NoTipQueja & $\begin{array}{l}\text { Carecer de un mecanismo o sistema de tipificación de quejas, } \\
\text { reclamos o sugerencias con las respectivas acciones de mejora. }\end{array}$ & Trato Digno \\
\hline NoTraslAfi & $\begin{array}{l}\text { Incumplimiento de la regulación relacionada con el traslado de } \\
\text { afiliados. }\end{array}$ & $\begin{array}{l}\text { Afiliación y representación } \\
\text { del Afiliado }\end{array}$ \\
\hline RetrAten & Falta de explicación por el retraso en la atención. & Trato Digno \\
\hline UPC $\$$ & $\begin{array}{l}\text { Incremento en los costos asociados a la incorporación de } \\
\text { tecnologías en las coberturas autorizadas por el POS que no son } \\
\text { asumidos por el Estado mediante una adecuada definición de la } \\
\text { UPC. }\end{array}$ & $\begin{array}{l}\text { Afiliación y representación } \\
\text { del Afiliado }\end{array}$ \\
\hline
\end{tabular}




\section{Referencias bibliográficas}

1. Banco Interamericano de Desarrollo. El estado de las reformas al Estado en América Latina. Lora E, editor. Washington, D. C.: Banco Mundial en coedición con Mayol Ediciones; 2007.

2. Congreso de la Republica de Colombia. Página web del Instituto Nacional de Salud [Internet]; 1993. Disponible en: https://www.ins.gov.co/normatividad/Leyes/LEY\%200100\%20DE\%201993.pdf

3. Gerschman S. Las reformas del Estado, de las políticas sociales y de salud. Un balance al final del Milenio. Cadernos de Saúde Pública. 1999;15(2): 293-302.

4. World Health Organization. The World health report 2000 - Health systems: improving performance. Ginebra: WHO; 2000.

5. World Health Organization. Everybody business: strengthening health systems to improve health outcomes: WHO's framework for action. Ginebra, Suiza: WHO; 2007.

6. Franco-Giraldo A. Sistemas de salud en condiciones de mercado: las reformas del último cuarto de siglo. Rev Fac Nac Salud Pública. 2014;32(1):95-107.

7. Ruíz-Gómez F. Friederich Ebert Stiftung en Colombia (Fescol). [Internet]. 2013 [citado 2017 jul. 13]. Disponible en: https://library.fes.de/pdf-files/bueros/kolumbien/09582-20130201.pdf

8. Ministerio de la Protección Social. Proyecto evaluación y reestructuración de los procesos, estrategias y organismos públicos y privados encargados de adelantar las funciones de vigilancia y control del sistema de salud. Bogotá: Ministerio de la Protección Social; 2008.

9. Saltman RB. Regulating incentives: the past and present role of the state in health care systems. Social Science \& Medicine. 2002;54(11):1677-84.

10. Ministerio de la Proteccion Social. Sitio web del Ministerio de Salud y la Protección Social. [Internet]. 2006 [citado 2015 mar. 13]. Disponible en: https://www.minsalud.gov.co/Normatividad/DECRETO\%201011\%20DE\%2 02006.pdf

11. Ministerio de la Protección Social. 1.er Informe Nacional de Calidad de la Atención en Salud. "INCAS Colombia 2009”. Bogotá: Ministerio de la Protección Social; 2009.

12. Congreso de la República. Sitio web de la Alcaldía Mayor de Bogotá. [Internet]. 2007 [citado 2015 oct. 2]. Disponible en: https://www.alcaldiabogota.gov.co/sisjur/normas/Norma1.jsp?i=22600.

13. Congreso de la República. Sitio web del Senado de la República de Colombia. [Internet]. 2011. Disponible en: ht tps://www.secretariasenado.gov.co/senado/basedoc/ley_1438_2011.html

14. Giuffrida A, Pinzón-Fonseca DA, Piñeros-García CA. Supervisión basada en riesgos, la nueva apuesta de la Supersalud. Monitor Estratégico. 2015;(7):8-16.

15. Balcom J, Pargaonker N. The road to risk-based supervision: Lessons from Chile. Journal of Securities Operations \& Custody. 2010; 3(3):228-40.

16. Eslava-Rincón J, Camelo-Tovar F, et al. Análisis interno y del entorno para definir los requerimientos del sistema de información para el monitoreo. Instituto de Salud Pública de la Pontificia Universidad Javeriana, Bogotá, 2015. Report N.o. Documento técnico GPGD 1755-15.

17. Corte Constitucional. Sitio web de la Corte Constitucional de la República de Colombia. [Internet]. 2008 [citado 2015 ago. 20]. Disponible en: https://www.corteconstitucional.gov.co/relatoria/2008/T-760-08.htm

18. Bernal-Acevedo O, Forero-Camacho JC. Sistemas de información en el sector salud en Colombia. Revista Gerencia y Políticas de Salud. 2011;10(21): 85-100.

19. HACCP. Hazard Analysis \& Critical Control Point Planning to Prevent the Spread of Invasive Species. Britton D, Heimowitz P, Pasko S, Patterson M, Thompson J, editores. Arlington: Hazard Analysis and Critical Control Point Planning for Natural Resource Management; 2011. 
20. Kendall KE, Kendall JE. Análisis y diseño de sistemas. Octava edición. México: Pearson Educación; 2011.

21. Serna LP. Prospectiva estratégica en la gestión del conocimiento: una propuesta para los grupos de investigación colombianos. Investigación y Desarrollo. 2013;21(1):237-59.

22. Ortegón E, Medina-Vásquez JE. Prospectiva: Construcción social del futuro. Santiago de Cali: ILPES-Universidad del Valle; 1997.

23. Daniels N. Reflective equilibrium. The Stanford Encyclopedia of Philosophy [Internet]. Stanford, Estados Unidos: The Metaphysics Research Lab, Center for the Study of Language and Information (CSLI), Stanford University; 2011. Disponible en: https://plato.stanford.edu/

24. Peñaloza RE, Mina L, Polania BE, Suelt V, González J, Ramírez D. Proyecto de evaluación de pertinencia y coherencia de las funciones y métodos de intervención regulatorias de inspección, vigilancia y control. Instituto de Salud Pública de la Pontificia Universidad Javeriana, Bogotá, 2015.

25. Suárez LF, Puerto S, Rodríguez LM, Ramírez J. La crisis del sistema de salud colombiano: una aproximación desde la legitimidad y la regulación. Rev Gerenc Polit Salud. 2017;16(32):34-50.

26. Tanahashi T. Health service coverage and its evaluation. Bull World Health Organ. 1978;56(2):295-303.

27. Martínez D. sitio web de la Pontificia Universidad Javeriana. [Internet]. 2008 [citado 2014 ago.]. Disponible en: $h$ ttps://www.javeriana.edu.co/redcups/METODOLOGiA_DEL_ANALISIS_SISTEMICO.pdf

28. Gold M. Beyond coverage and supply: measuring access to healthcare in today's market. Health Services Research. 1998;33(3):625-84.

29. Eslava-Rincón JI, Camelo-Tovar FA, Mejía-Rocha MM, Mina-Rosero L. Sistema de monitoreo de intervenciones regulatorias y de inspección, vigilancia y control en el Sistema General de Seguridad Social (SIMIV): Una propuesta basada para la gestión de riesgos para la IVC territorial. En: Eslava-Rincón JI, Camelo-Tovar FA, Acosta-Ramírez NA, Vivas-Tobar C I, Mateus-Solarte JC, Pineda-Granados FY. Modelo integrado para la inspección, vigilancia y control de riesgos de acceso, uso y calidad de los servicios de salud, en el ámbito territorial. Bogotá: Fundación para la Educación y el Desarrollo Social; 2018. p. 292-360.

30. Cuellar CM, Montaño JI, Morales LA, Mesa DC, Palacios PM, Llano A, Trujillo AA. Guía metodológica nacional para la caracterización de fuentes de información para la salud en Colombia. MinSalud. 2014 [citado 2017 jul. 10]. Disponible en: https://www.minsalud.gov.co/sites/rid/Lists/BibliotecaDigital/RIDE/VS/ED/GCFI/gui a\%20definitiva\%2019062014.pdf

31. Gañan-Echavarría JL. Los muertos de Ley 100. Prevalencia de la libertad económica sobre el derecho fundamental a la salud: una razón de su ineficacia Medellín: Universidad de Antioquia; 2013.

32. Rivadeneira AG. Marco conceptual y legal sobre la gestión de riesgo en Colombia: Aportes para su implementación. Monitor Estratégico. 2014;(5):4-11.

33. Ovies DP, Agudelo SM. Lecciones aprendidas en la implementación de sistemas nacionales de información de salud interoperables: una revisión sistemática. Rev Panam Salud Publica. 2014;35(5/6):415-23.

34. World Health Organization. Monitoring the building blocks of health systems: a handbook of indicators and their measurement strategies. Ginebra: WHO; 2010.

35. World Health Organization. Monitoring and evaluation of health systems strengthening: An operational framework. Ginebra: WHO; 2009.

36. Bernal-Acevedo O, Forero-Camacho JC, Hernández LJ, León LJ. Sistemas de Información en el Sector Salud en Colombia. En: Bernal-Acevedo O, Gutiérrez C. La salud en Colombia: logros, retos y recomendaciones. Bogotá: Ediciones Uniandes; 2012. p. 480-3.

Licencia Creative Commons CC BY 4.0

Para citar este artículo: Eslava-Rincón JI, Camelo-Tovar FA, Mina-Rosero L, Vásquez-Candia ME, MejíaRocha MM. Análisis de la capacidad de respuesta de los sistemas de información en salud para la supervisión de riesgos que afectan el derecho a la salud en Colombia. Rev. Gerenc. Polit. Salud. 2018;17(35). https://doi.org/10.11144/Javeriana.rgps17-35.acrs 